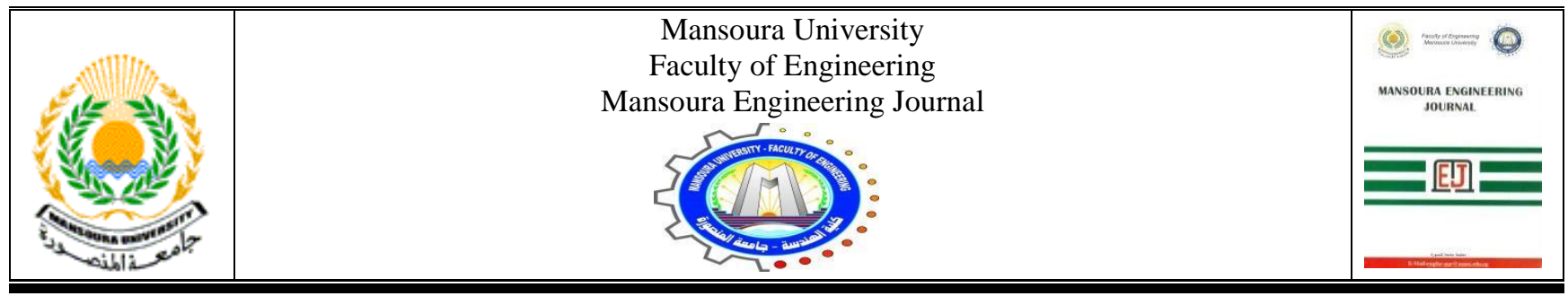

\title{
Extrapolate the role of urban spaces and methods of development in the era of information technology (Case study of urban spaces within the Smart Cities)

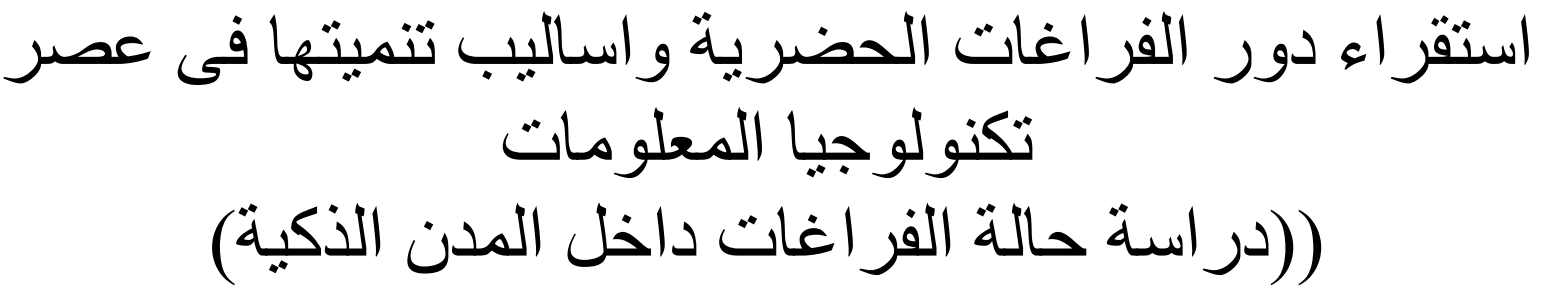

Ahmed Helmy Nabil and Rania Badawy Shokry

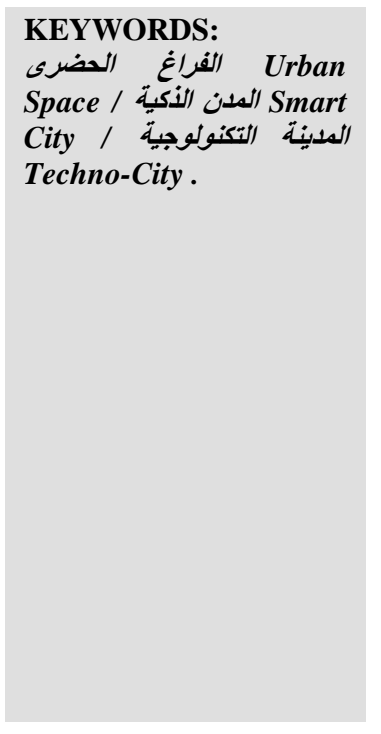

\begin{tabular}{l} 
Abstract- Urban Spaces is a true mirror of the conditions of economic, social, \\
cultural and political community they represent the focal points of public life and the \\
exercise of various activities in addition to its role in giving a distinguished architectural \\
style of the city and each had a vacuum past his own personality, which meets the needs of \\
its users, but that over time and as a result of the rapid development in lifestyle It \\
disappeared role within the urban fabric of the city as the site of the interaction between \\
man and his environment. \\
Urban places are not only built area, but also include activities that users are given the \\
goals of the space distinctive character in terms of size and function. Finding the entrance \\
to the development of the Urban space in line with the local environment will return \\
positive returns positive impact on the urban development of the city, and methods of \\
urban use of information technology development or the so-called "Smart City Smart city" \\
which aims to create sustainable and economic development, high quality of life, and are \\
considered infrastructure for information and communication technology have a nervous \\
system, and the organization of the interactions between components. \\
But most of the establishment of smart cities initiatives focused on the use of \\
technological applications in the transportation and delivery of services and urban \\
management areas, but it did not address enough to use these applications in the design of \\
urban spaces smart cities, so research aims to recognize the importance of smart urban \\
spaces in urban infrastructure of the city and extrapolation of its role in the era of \\
information technology. And perceive the importance and extent of work to be \\
distinguished, respected and enriched in line with the current and future needs of its users. \\
\hline
\end{tabular}

الملخص العربي:- تعتبر الفراغات العمرانية المرآة الحقيقية لأحوال المجتمع

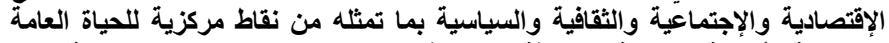

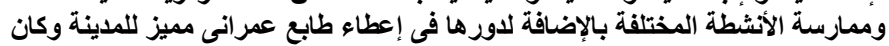

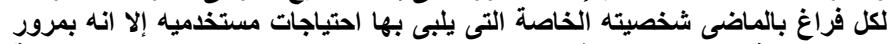

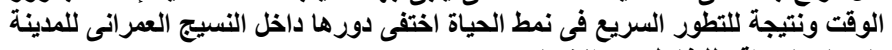

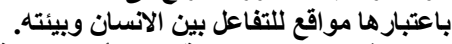

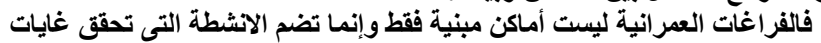

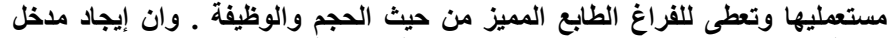
لتنمية الفراغ العمرانى بشكل يتوافق مع البيئة المحلية سيعود بمردود إيجابى على المئي
Received: (1 February, 2016) - revised: (19 April, 2016) - accepted: (4 May, 2016)

Ahmed Helmy Nabil, Assistant Lecturer of urban planning \& urban design, Department of Architecture - Faculty of Engineering - Egyptian Russian University.

Rania Badawy Shokry, Lecturer of urban planning \& urban design, Department of Architecture - Faculty of Engineering - Egyptian Russian University 
عناصر مساعدة من مقاعد و أماكن مظلله.

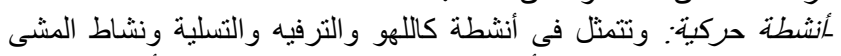

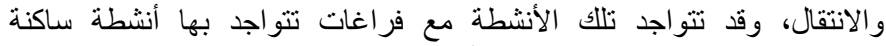

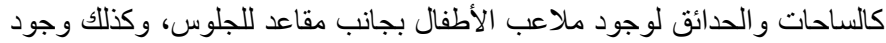

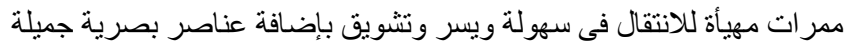

2-3 الأهبية البيئبة

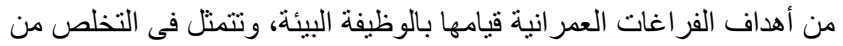

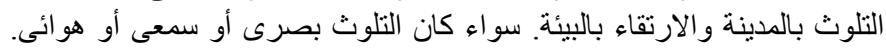

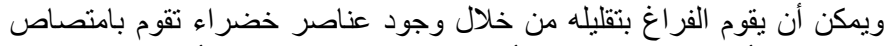

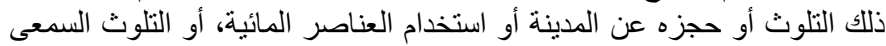

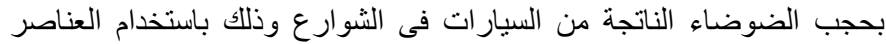
الخضر اءه و المائية.

3-3 - - 3 الأهية الاجتماعية

يتم فى الفراغ الخارجى معظم الجوانب الحئه الحياتية، ويعد هو المتتفس للمدينة،

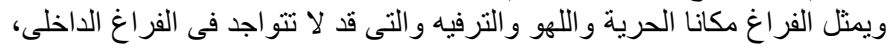
بجانب الاحتكاك مع المجتمع المحيط.

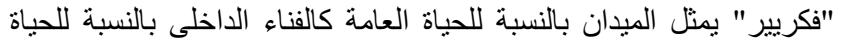

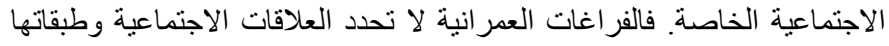

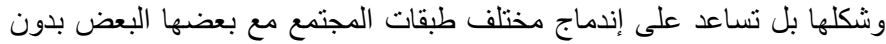

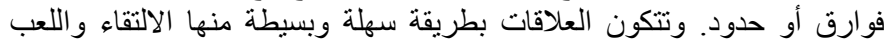

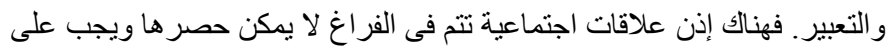

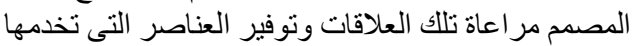

4-3 أهمية نفسية

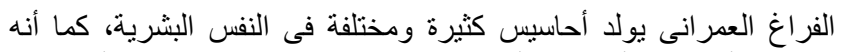

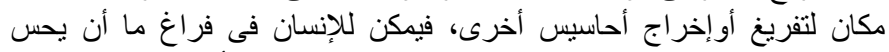

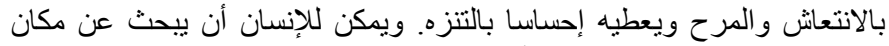

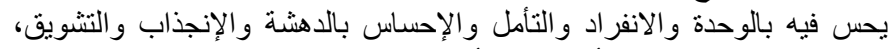

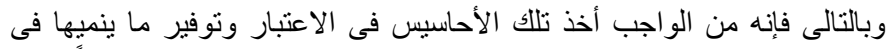

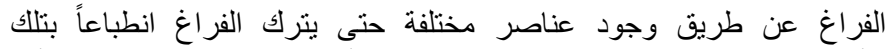

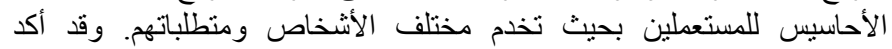

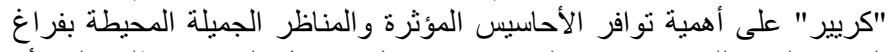

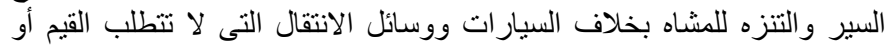

المناظر الجميلة

5-3 أهمبة اقتصادية

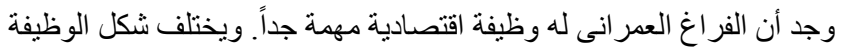

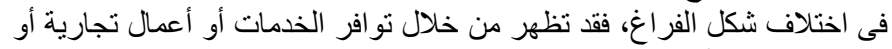

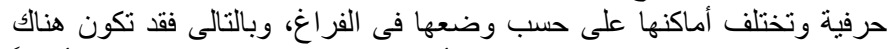

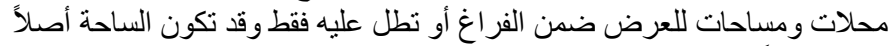
تمثل فراغاً للسوق مع نوفير خدمات للمستعملين كمقاعد للجلوس أو الو الراحة و استكمال الثراء فاء

6-3 أهمبة سباسبة

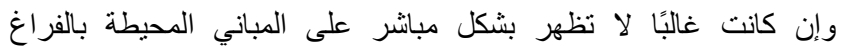

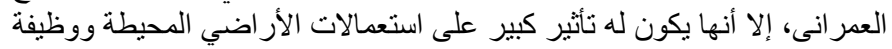
الفراغ بشكل عام ونو عية الأنشطة المقامة داخله.

\section{معايير تصميم الفراغات الحضرية .IV}

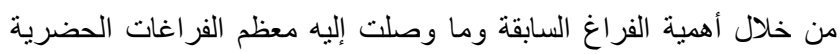

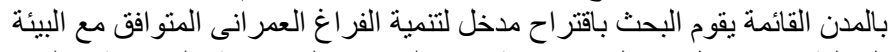

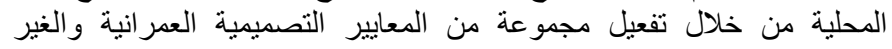

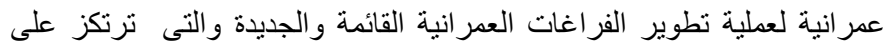

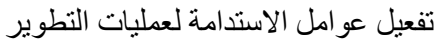

التنمية العمرانية للمدينة، ومن اساليب تنمية المدن استخدام تكنولوجيا المعلومات او ما

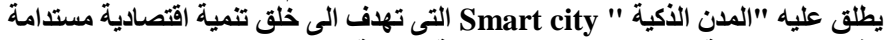

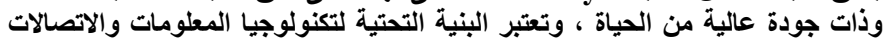

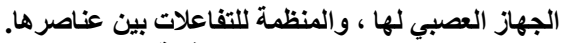

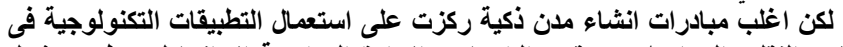

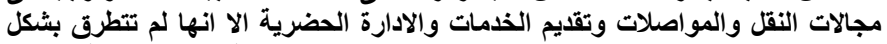

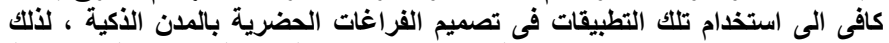

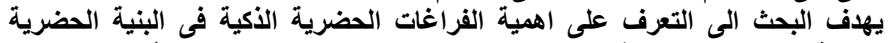

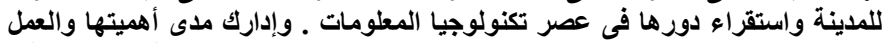

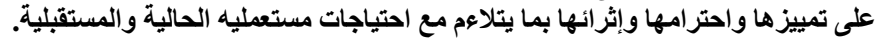

\section{I}

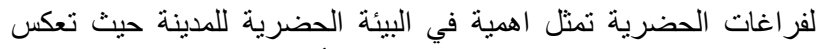

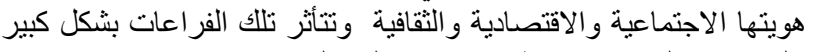

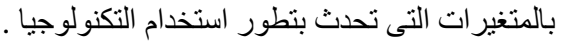

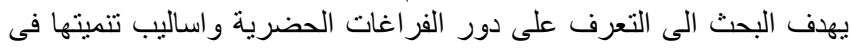

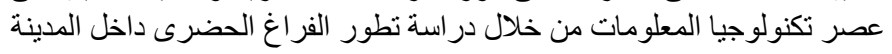

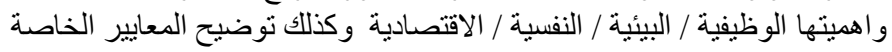

وكذلك نوضيح مفهوم المدن الذكية واهمية الفراغات الحضرية الذكية بالمدينة

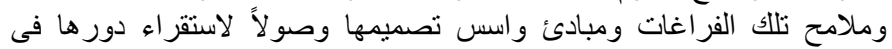
عصر تكنولوجيا المعلومات

\section{II أهمية البحث}

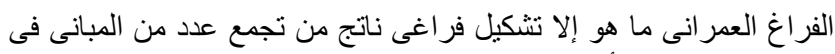

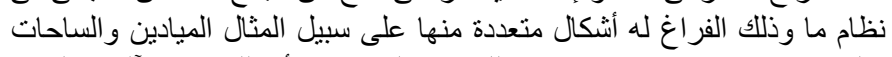

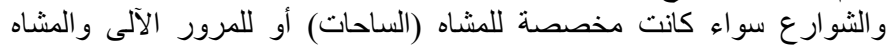
(الميادين.) (الموعل)

وتشكل الفراغات العمرانية أهمية كبيرة داخل المدينة لما تمثله من قيمة

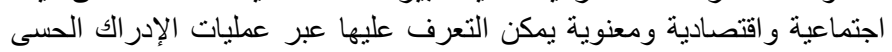

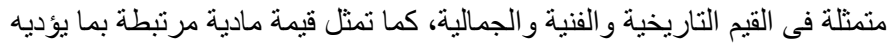

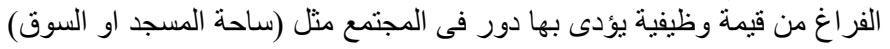

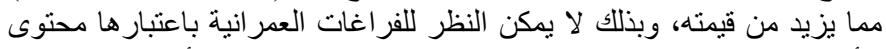

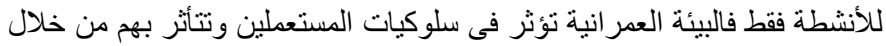

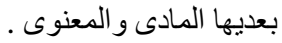

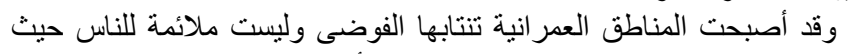

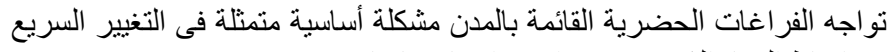

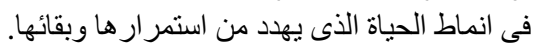

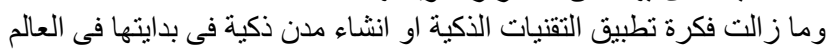

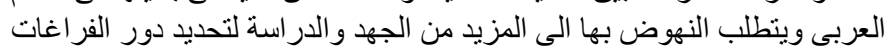

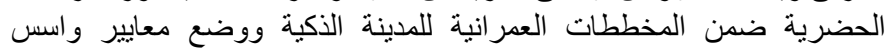
تصميمية لها كفر اغات حضرية ذكية فى ظل تغير انماط احتياجات مستخدميها الاجتماعية و الثقافية و الاقتصادية

\section{أفراغات الحضرية واهميتها فى المدينة .III}

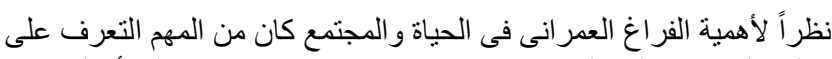

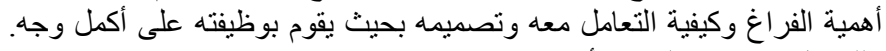

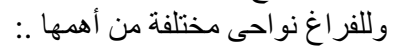

1-3 الأهية الوظيفية

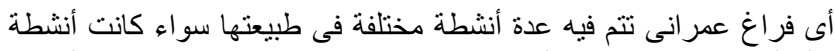

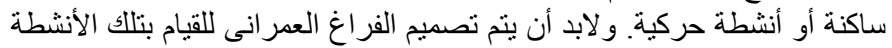

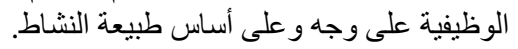

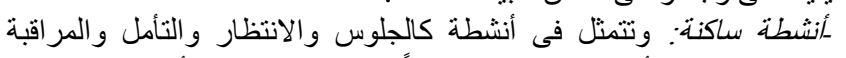
والاسترخاء. ويجب أن يكون الفراغ مهيئًا للقيام بمثل تللك الأنشطة من نوفر 
• أن يتسع الطريق لأونتوبيسات الرحلات السياحية فى الفر اغات الرئيسية

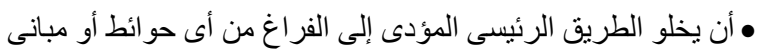

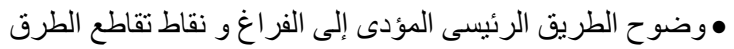

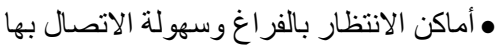

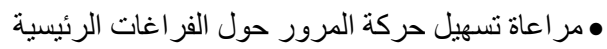

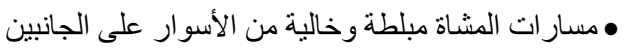

$$
\text { 3-4-4 عناصر الفرش }
$$

• أحتو اء الفراغ على عناصر شبه ثابتة (فرش) ثُدِّم النشاط الذي سيُمارس

• • ماكن وضع اللوحات الارشادية و اللاّفتات وكيفية تصميمها

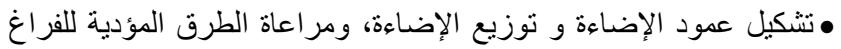
وكمبة الإضاءة اللازمة

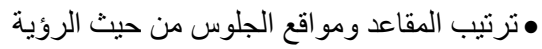

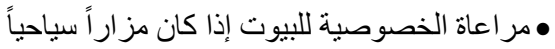

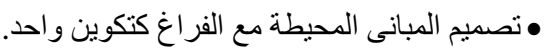

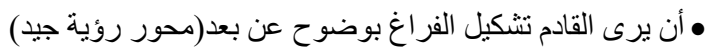

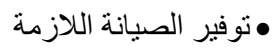
• سهولة التغيير للتالف وخاصةً لعناصر الإضاءة

\section{SMART URBAN SPACEEية الفراغات الحضرية الأكية .V}

تعتبر العلاقة بين العمران وتكنولوجيا المعلومات والاتصالات مinformation \& Telecommunication Technology (ICT) الموضو عات الهامة والمؤثرة على شكل وبنية عمر ان المدينة ، وقد تز ايد الاهتمام

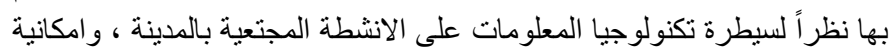

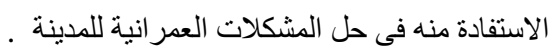

$$
\text { 1-5 مفهوم الددن الذكبة }
$$

لا يوجد تعريف محدد لمفهوم المدن الذكية Smart City الا ان بداية استخدام

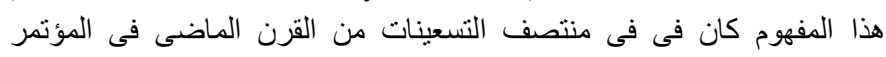

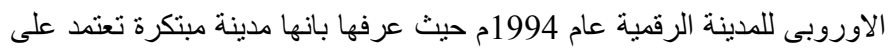

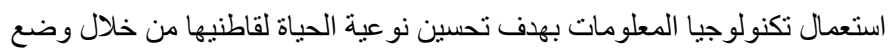

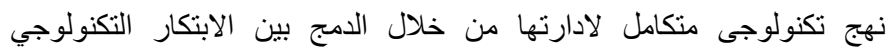

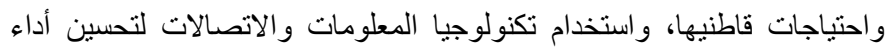
قطاعات الخدمات و المر افق بنتلك المدن.

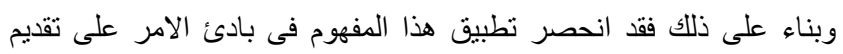

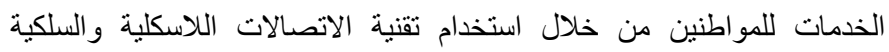

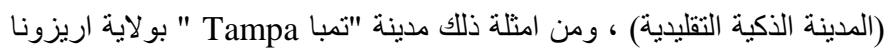

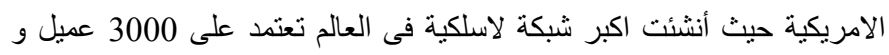

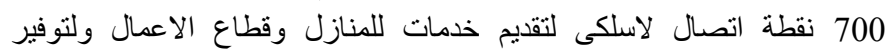

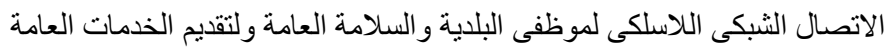

$$
\text { لجامعة ولاية اريزونا }
$$

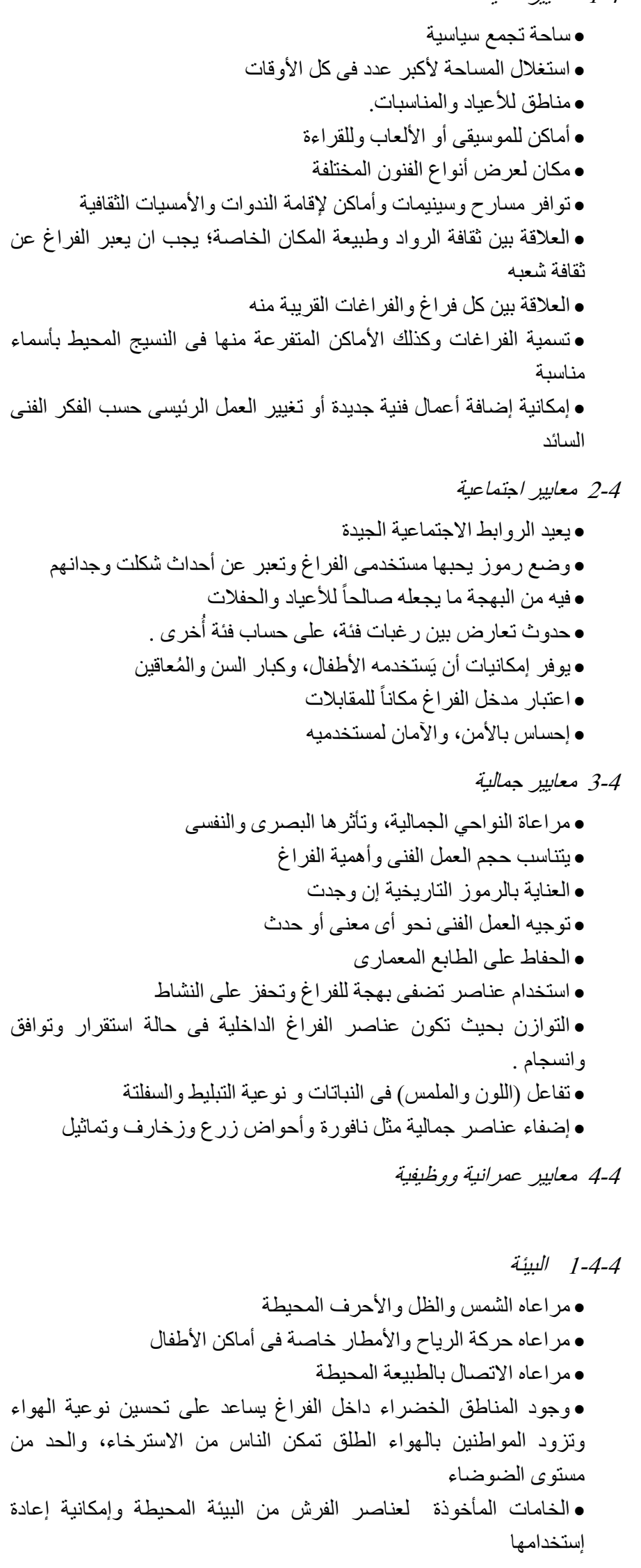




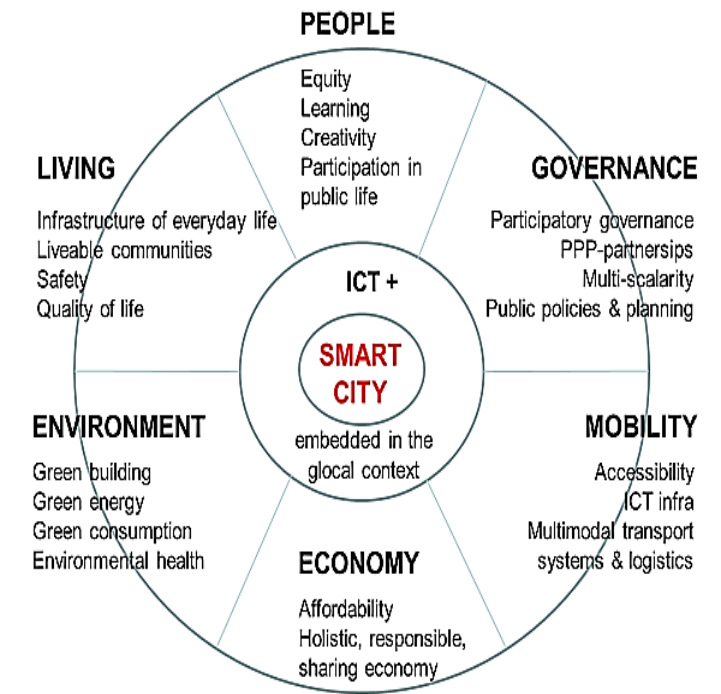

شكل (2) خصائص المدن الذكية

Characteristics of a Smart City - Smart Cities Framework for : المرجع الند Pakistan - 2012

2-5 ملامح الفراغات الحضرية الذكبية

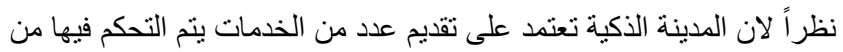

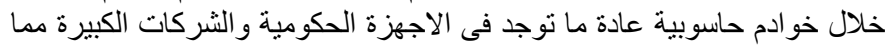

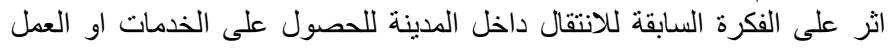

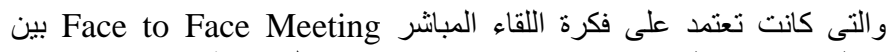

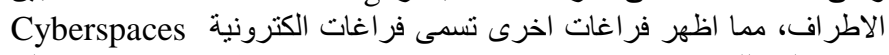

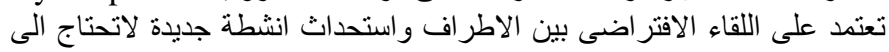

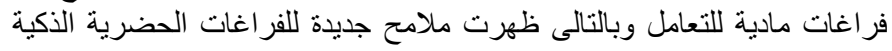

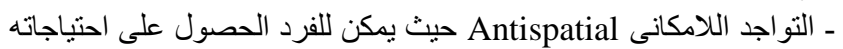

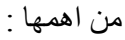

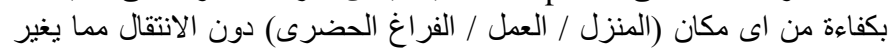
الاهمية المكانية التقليدية للعلاقات الونز الوظيفية للمدينة.

- اللاتزامن Asynchronous حيث يمكن للافراد و المؤسسات المختلفة

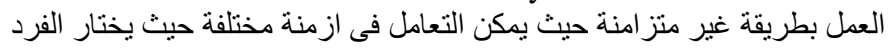
الوقت المناسب للحصول على على احتياجاته.

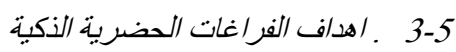

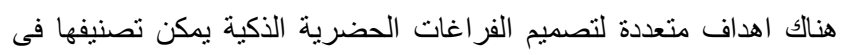

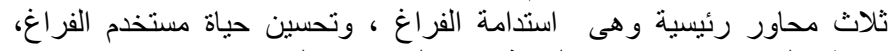

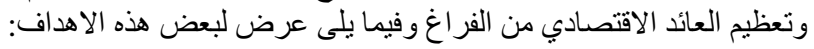
ل

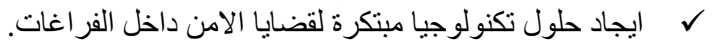

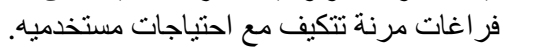

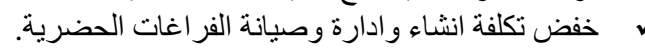

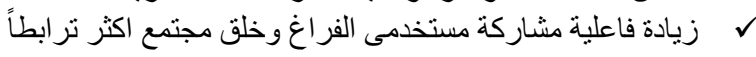

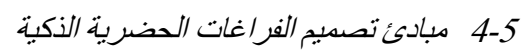

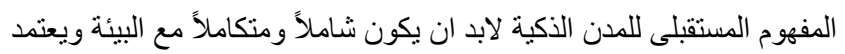

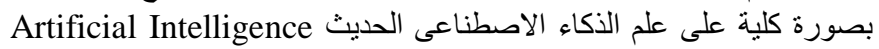

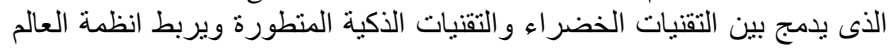

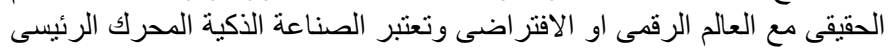

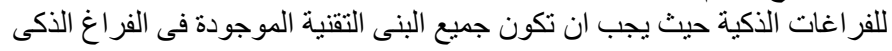

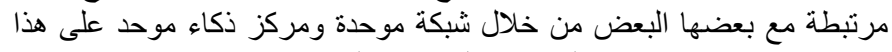

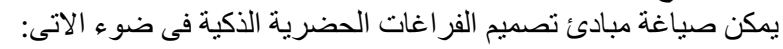

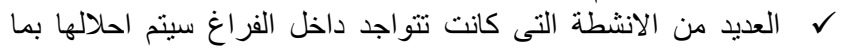
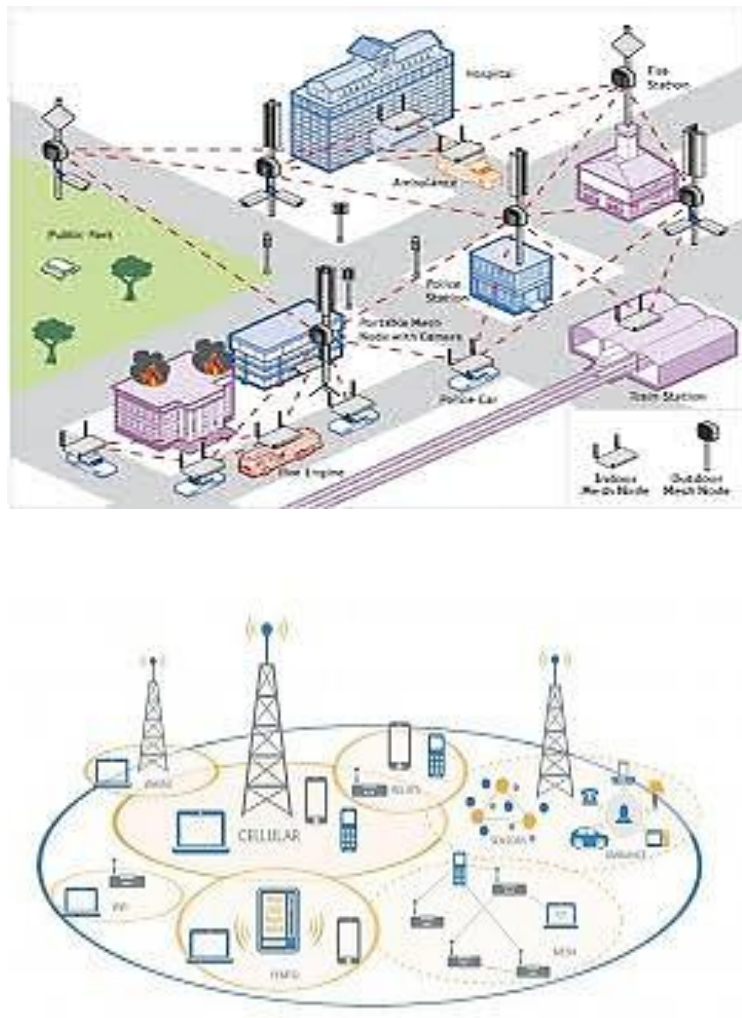

شكل (1) فكرة استخدام شبكة اتصال اللاسلكى

nterDigital Intelligent Wi-Fi Offload Solution Highlighted to Top Operators at Broadband World Forum - www.interdigital.com

ثم تطور المفهوم الى المدن المعرفية الذكية وهي المدن التي تستهدف التنمية

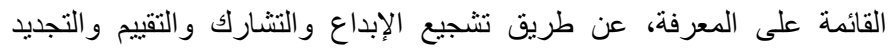

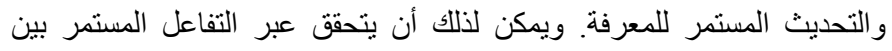

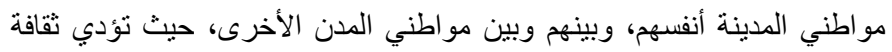

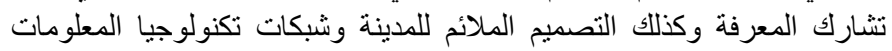

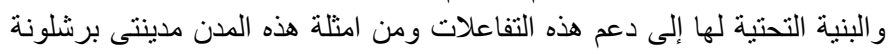

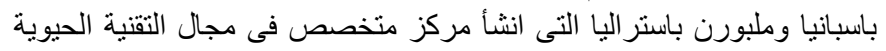
Biotechnology

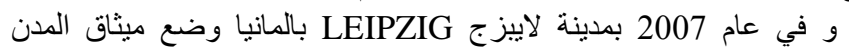

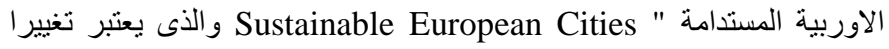

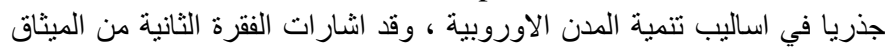

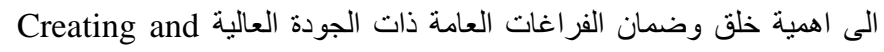
ensuring high-quality public spaces

كما وضع الاتحاد الأوروبي اطار برنامج "أفق 20202020 "Horizon

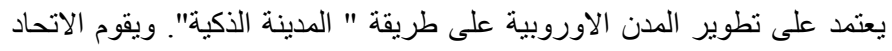

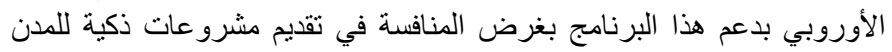

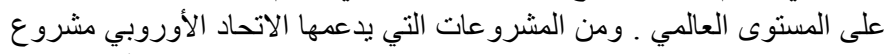

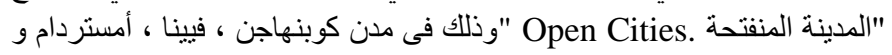
برلين.

وتتميز خصائص تلك المدن الذى تعتمد على الاقتصاد الذكى Smart

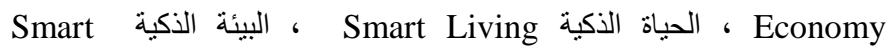

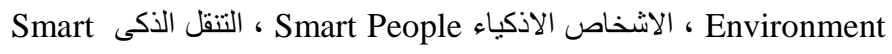
Smart Governance Mobility 


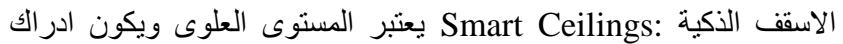

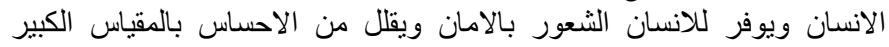

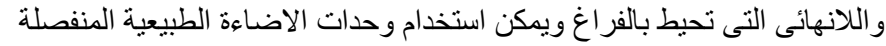

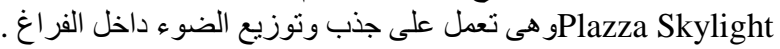

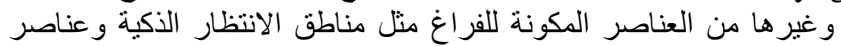

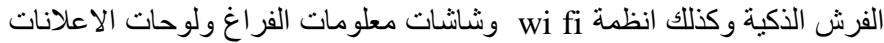
التى توفر الراحة والامان لمستخدمى الفراغ النغان

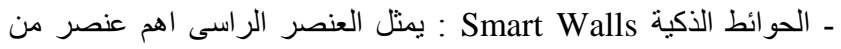

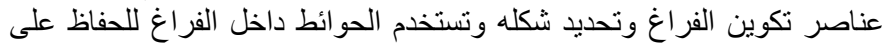

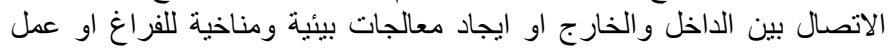

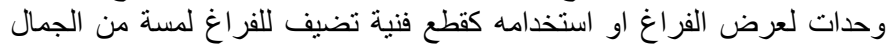

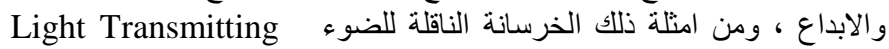

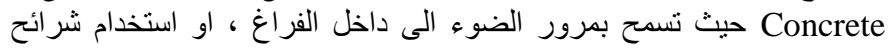

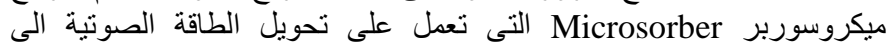

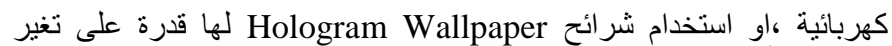

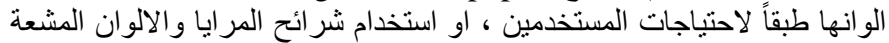
D Radiant Color and Mirror Film3

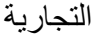

\section{VI}

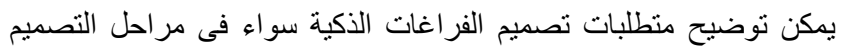

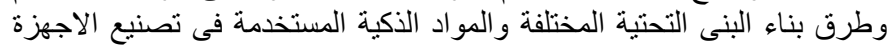

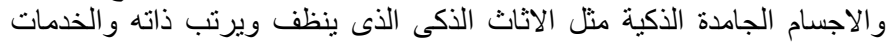

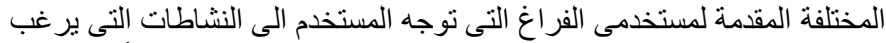

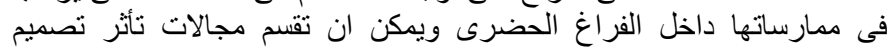

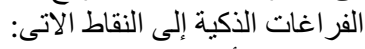

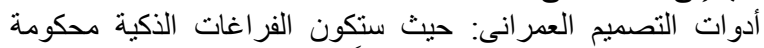

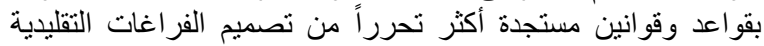

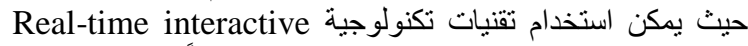
Interactivity ويكون لمستخدى الفراغ اكثر تفاعلاً

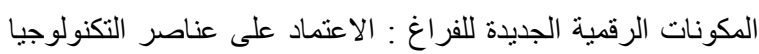

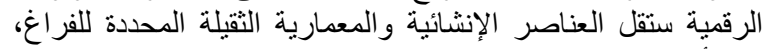

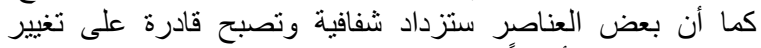
صفاتها بما يحقق أهدافاً بيئية وتثكيلية وتكنولونية ونية

المنطلبات والتحولات الوظيفية للفراغات : في عصر تكنولوجيا

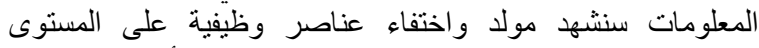

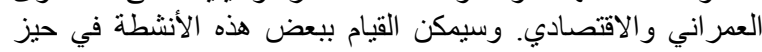
فراغي غير مادي بال "Cyber City" :المدينة ذات الات التحكم

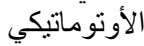

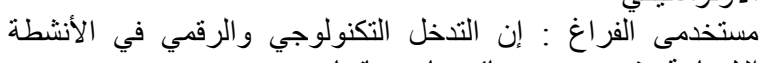

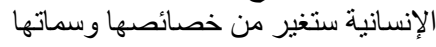

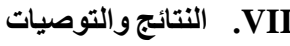

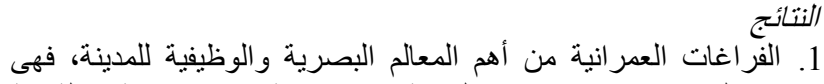
تعكس العديد من خصائص المدينة الاقتصادية والاجتماعية والعية والثية الثقافية و الحضارية بل والسياسية أيضا، وتثنهر معظم الددن العالمية بالفراغات

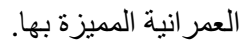
2. الفراغات العمرانية من الأماكن الهامة في البيئة الحضرية التي تعكس

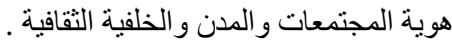

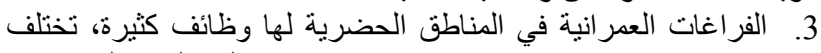

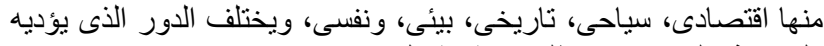
كل فر اغ على حسب منطلبات وثقافة كل شعب.
يماثلها من الفراغات الافتراضية (مثل امكانية التسوق او التعلم من خلال

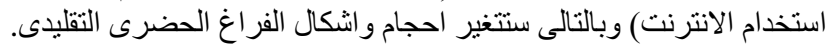

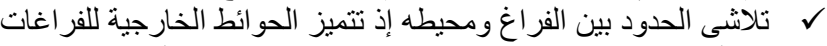

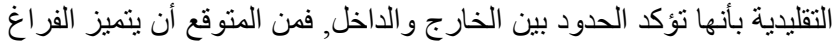

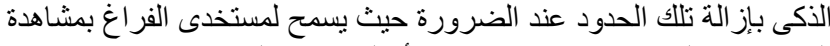

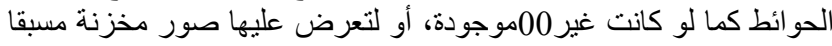

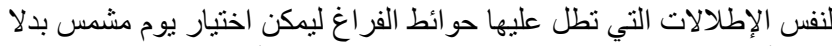

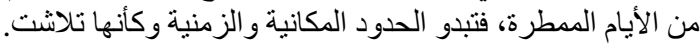

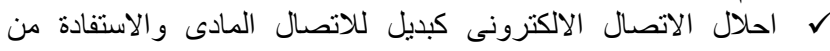

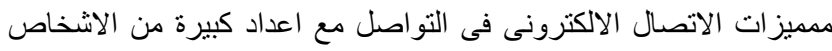
في اوقات مختلفة. ل الاستفادة من التقنيات الالكترونية في خلق بيئات عمر انبة اكثر ملائمة

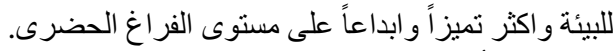

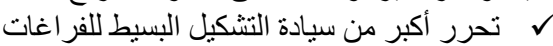

ل التخلي عن التوحيد القياسي حيث لن لن يكون التوحيد القياس

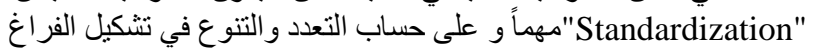

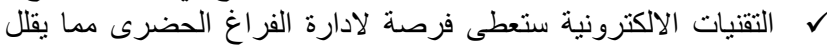

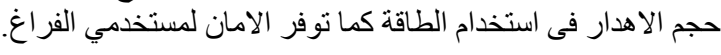
5-5 مفردات الفراغات الحضرية الذكية يمكن تمييز مفردات الفر اغات الحضرية الذية الذكية كالتالى:

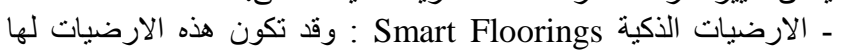
قدرة على انتاج الطاقة الحركية وتحويلها الى طاقة كهربائية او ما بطلق على عليه Energy Floors

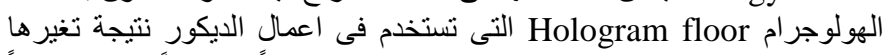

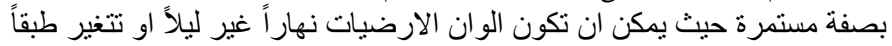

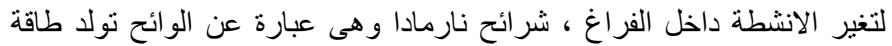

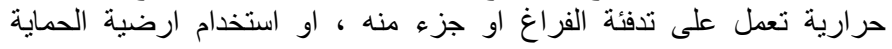

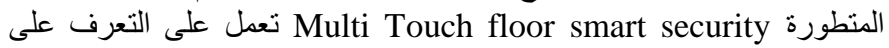
الثخص الو اقففوقها من بصمة القدم ويمكن استخدامها لتأمين مداخل الفر الغ

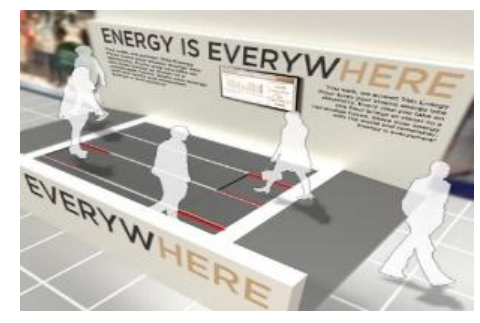

الارضيات المنتجة للطاقة Energy Floors

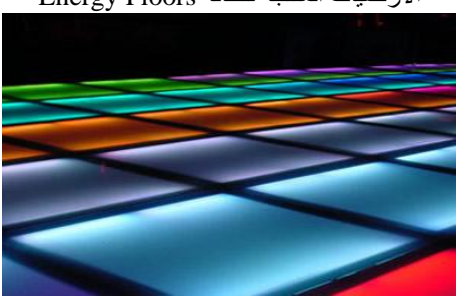

بلاطات الهولوجرام Hologram floor

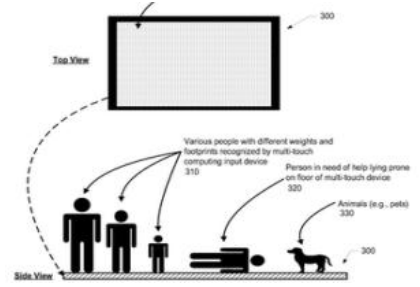

Multi Touch floor smart ارضية الحماية المنطورة security Smart Floorings شكل (3) الارضيات الذكية 
7. اضافة معاييرومبادئ تصميم الفراغات الذكية مثل استخدام تقنيات تكنولوجية Real-time interactive components تجعل مستخدمى ترئي الفراغ اكثر تفاعلاً معه بقانون 119 "البناء الموحد"

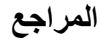

ماريان يسرى،2010م, رسالة ماجستير" التغير والتحول فى الميادين بالقاهرة"،كلية الهنسة

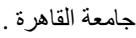

كرانيا بدوى، المطرية 2010م، رسالة دكتوراه " منهجية التصميم الحضرى للمبادين الكبرى بمصر" كلية الهندسة بالمطرية جامعه حلوان.

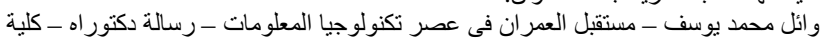

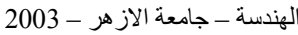

فاطمة عبد الرحمن باعثمان - اهمية التصميم لجعل مكة المكرمة اكثر مدن العالم ذكاء ـ مجلة

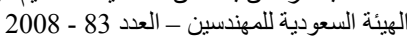

[5] http://ec.europa.eu/regional_policy/archive/themes/urban/leipzig_charte .pdf

[6] https://ec.europa.eu/programmes/horizon2020/h2020-sections

[7] http://www.opencities.net/

[8] http://smartcitiescouncil.com/

[9] http://www.smart-cities.eu/benchmarking.html

[10] نهى محمد عز الدين - التقتيات الذكية المستخدمة في ترشيد استهلاك الطاقة داخل فراغات

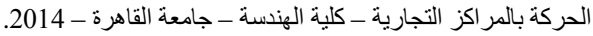

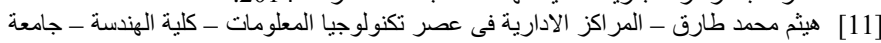
القاهرة - 2005

[12] Comparative Study of Smart Cities in Europe and China 2014 - China Academy of Information and Communications Technology • EU-China Policy Dialogues Support Facility II - August 2014

[13] Smart Rules for Smart Cities - Managing Efficient Cities in EuroMediterranean Countries - Eleonora Riva Sanseverino - 2014

[14] E-TOPIA "Urban life, Jim-but not as we know it" - William J. Mitchell - Cambridge, MA: The MIT Press, 1999

[15] Adam,Ritchie\&Randall,Thomas.(2008)" Sustainable Urban Design"An Environmental Approach", by Taylor \& Francis .2nd Edition, 16th December

[16] Chris van Unfallen,(2012) "Urban Spaces: Plazas, Squares \& Streetscapes" (Architecture in Focus) December 16, 2012

[17] Bear, Anne, (1990) "Environment Planning for Development", and an imprint of Chapman, England.

[18] Catherin Bull, Davisi Boontharm, Claire Parin, Darko Radovic, Guy Tapie,(2007)"Cross-Cultural Urban Design" .26th November by Routledge

[19] Cooper,C. and Francis,c.(1990),"People places - Design guidelines for Urban open Spaces", Van Nostr and Reinhold, New york,1990.

[20] Jordan hill, (2003), Inclusive urban design "Street for life "oxford ox 2 wheeller rood.

[21] Moughtin, Cliff, (2003). "Urban Design: street and square". London: Butterworth, ISBN 0750604166. Routledge; 3 edition (June 19, 2003).

[22] Wang Shu YiYi, (2002)" Urban Design - Street and Square" (Second Edition) (Traditional Chinese Edition) by Paperback - January 1

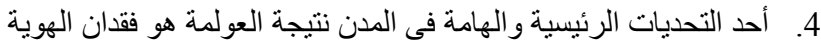

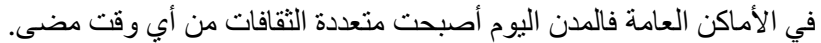

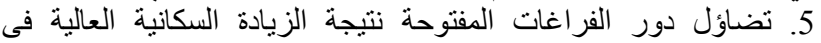

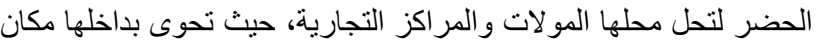

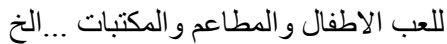

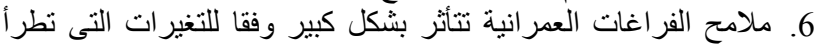

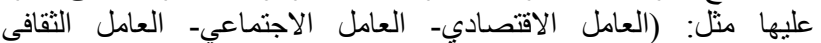

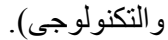

7. تقنيات الاتصالات و الالكترونيات لها تاثيرها الواضح على اضلى العملية

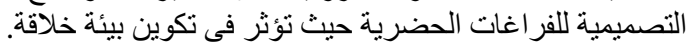

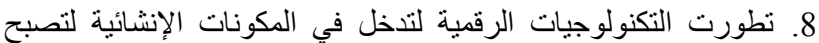

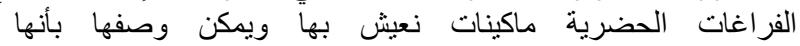
" Artificial "الفراغ الآلي و و "Instrumental Space " والذكاء الاصطناعي. Intelligence"

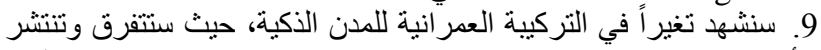
الأنشطة الإنسانية، وستبتعد الإدارات عن فئن موقع الإنتاج. وسيمكن القيام

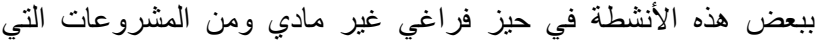

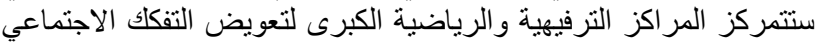

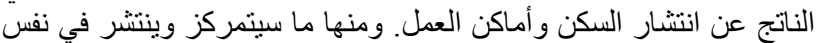

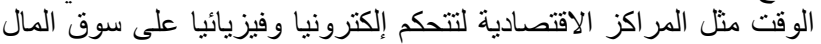

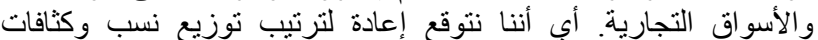

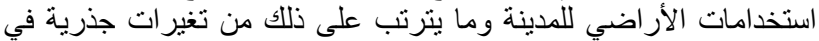

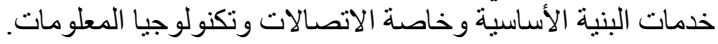

2 2.7

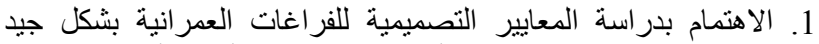

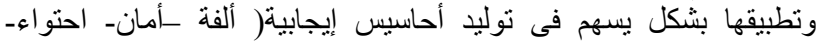

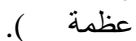

2. يجب التوجه إلى إحباء وثقل دور الفراغات العمرانية حبث تضاءل

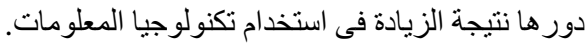

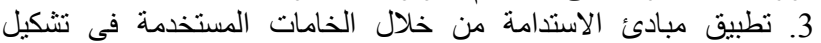
العناصر المختلفة للفراغ العمرانئ المئ والمأخوذة من البيئة المحيطة لإمكانية

إعاداة إستخدامها العزئ

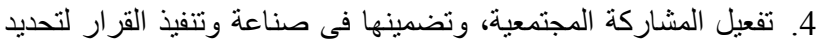

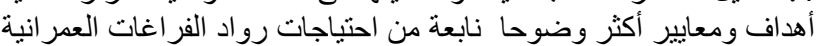
للتطوير

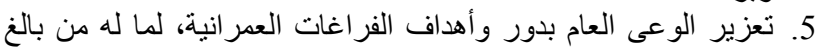

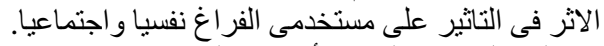

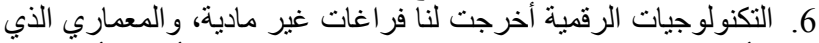

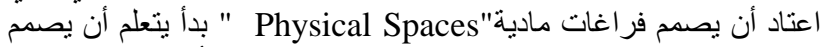

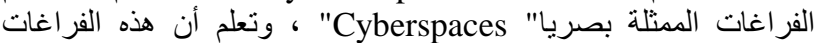
محكومة بقواعد وقوانين مستجدة أكثر تحررا من تصميم الفرات اغات المادية، مما فتح أمامه مجالات فكرية جديدة. 